\title{
Territorialités et aires marines protégées à Moorea (Polynésie française)
}

\section{Cécile Gaspar et Tamatoa Bambridge}

\section{(2) OpenEdition \\ 1 Journals}

\section{Édition électronique}

URL : http://journals.openedition.org/jso/2462

DOI : $10.4000 /$ jso.2462

ISSN : $1760-7256$

\section{Éditeur}

Société des océanistes

\section{Édition imprimée}

Date de publication : 15 décembre 2008

Pagination : 231-246

ISBN : 978-2-85430-012-3

ISSN : 0300-953x

\section{Référence électronique}

Cécile Gaspar et Tamatoa Bambridge, «Territorialités et aires marines protégées à Moorea (Polynésie française) », Journal de la Société des Océanistes [En ligne], 126-127| Année 2008, mis en ligne le 15 décembre 2011, consulté le 16 juin 2020. URL : http://journals.openedition.org/jso/2462 ; DOI : https://doi.org/10.4000/jso.2462 


\title{
Territorialités et aires marines protégées à Moorea (Polynésie française)
}

\author{
par
}

Cécile GASPAR* et Tamatoa BAMBRIDGE**

\section{RÉSUMÉ}

Cet article étudie les dynamiques territoriales liées à la mise en place d'aires marines protégées dans le lagon de Moorea. L'instauration de ces zones spécifiques implique un double processus, de " déterritorialisation " (consistant à quitter un espace lagonaire avec le cadre analytique ou " mental » de référence qui lui est associé, qu'il soit écologique, technologique ou social) et de " reterritorialisation »-consistant à aménager un nouveau rapport à un territoire marin et à intérioriser de nouvelles références culturelles et les pratiques qui en découlent (Deleuze et Guattari, 1980). Cette double dynamique est historique, induite par des événements antérieurs se plaçant dans l'évolution d'une population ou d'un territoire. Cet article montre la nature des enjeux qui se nouent autour de la création d'aires marines protégées et les logiques d'acteurs (État, gouvernement, communauté de pêcheurs, acteurs hôteliers, personnels d'animation, touristes) qui perdurent ou émergent.

MotS-CLÉS : aires marines protégées, Moorea, lagon, tourisme, pêche, plan de gestion de l'espace maritime (PGEM)

\section{Présentation du sujet}

\section{Problématique générale}

La littérature récente concernant les aires marines protégées est d'autant plus abon-

\begin{abstract}
This study contributes to the analysis of the territorial consequences of the set up of Marine Protected Areas (MPA) in the lagoon of Moorea Island. The basic hypothesis is that Marine Protected Areas include two different phases of a continuous process: "deterritorialisation» (leaving not only the lagoon space, but also mental references associated to it: ecological, technological and social), and «reterritorialisation»- developing a new relationship with a territory, and internalizing new cultural and practical references which go with it (Deleuze et Guattari, 1980). The progress of these concepts is historical and related to past events involved in territorial issues. This paper highlights the nature and the evolution of stakes between the actors involved in the set up of marine protected areas (state, government, fishermen communities, hotelltourism actors).
\end{abstract}

KeYwORds: Marine Protected Areas, Moorea, lagoon, tourism, fishery, territoriality

dante que la communauté internationale s'est imposé des objectifs ambitieux en ce qui concerne la protection des surfaces maritimes côtières ${ }^{1}$. En Polynésie française, dotée d'une surface maritime de près de 5 millions de $\mathrm{km}^{2}$,

1. Le Sommet mondial pour le développement durable, qui s'est tenu à Johannesburg en 2002, a réaffirmé le rôle des aires marines protégées dans la conservation de la biodiversité, en fixant pour objectif la mise en protection de 20 à $30 \%$ de la surface maritime à l'horizon 2012, évaluée à environ $2 \%$ actuellement.

* Présidente de l'association Te mana o te moana, docteur vétérinaire, Moorea, cecile.gaspar@gmail.com

** Chargé de recherche au cnrs (UMR 5244 EPHE-CNRS-université de Perpignan), tamatoa@univ-paris1.fr 
seuls les lagons de Fakarava (atoll de l'archipel des Tuamotu) et de Moorea (îles de l'archipel des îles du vent, proche de Tahiti) sont protégés par une réglementation instaurant des aires marines protégées $^{2}$ (AMP).

Malgré l'importance des enjeux humains et environnementaux concernés par la mise en place des AMP, les auteurs qui s'interrogent sur la « territorialisation » détruite et reconstruite par la mise en place des AMP sont rares. À tel point que certains se questionnent sur le statut territorial de l'AMP, indiquant qu'elle serait « un territoire en mal de reconnaissance » (David et Thomassin, 2007).

En effet, les AMP sont non seulement le produit d'évolutions historiques et socioéconomiques locales précises mais elles induisent aussi un nouveau rapport au territoire. C'est la double dynamique de cette transformation, dérivée d'un état antérieur et reconstruite sur des bases nouvelles, qui est l'objet de ce travail.

Le but de cette recherche est d'analyser, sous l'angle des conséquences qu'il a eues sur l'environnement matériel et humain, le plan de gestion de l'espace maritime (PGEM) mis en place dans le lagon de Moorea (4 900 hectares). L'exemple de Moorea nous conduira à reconnaître la pertinence d'une approche socio-anthropologique pour traiter la question de la mise en place des AMP dans les pays du Pacifique Sud. Nous prenons en compte, dans cette approche, certains aspects souvent oubliés ou simplifiés de ces processus, et notamment les points suivants :

- Dans l'ensemble du Pacifique, les objectifs environnementaux sont souvent mis en avant pour justifier la mise en place d'une AMP, alors même que celle-ci a un impact fort sur les populations insulaires locales et leurs activités.

- La mise en place d'aires protégées n'est historiquement pas neutre. Elle s'inscrit dans une trajectoire historique et des dynamiques socioéconomiques que les AMP contribuent à interrompre, détourner ou, au contraire, à accentuer.

- Dans le même temps, à la mise en place de l'AMP, planifiée par l'administration, s'ajoutent des effets induits, résultant à la fois des difficultés rencontrées lors de la mise en œuvre du PGEM, de l'organisation sociale des groupes considérés et de la marge de manœuvre de l'administration vis-à-vis des autorités et des acteurs locaux.

\section{Cadre conceptuel}

En mettant en place un PGEM à Moorea, l'administration a cherché à protéger les écosystèmes coralliens et à faciliter l'application d'une politique de développement durable au bénéfice des populations qui en dépendent. Ce faisant, l'administration participe à un double processus. D'une part, elle interrompt ou renforce, selon les cas, des dynamiques socio-historiques existantes. D'autre part, elle participe à l'émergence de nouveaux rapports au sein du territoire.

La mise en place d'aires marines protégées, comme à Moorea, implique successivement une déterritorialisation (consistant à quitter un espace lagonaire avec le cadre « mental » de référence qui lui est associé, qu'il soit écologique, technologique ou social, tel que la coutume l'avait édifié) et une reterritorialisation (conduisant à y aménager de nouveaux rapports matériels avec le territoire marin mais aussi à y intérioriser de nouvelles références mentales et culturelles) (Deleuze et Guattari, 1980) ${ }^{3}$.

Cette double dynamique est fondamentalement plurielle : toutes les déterritorialisations ne s'opèrent pas sur les mêmes schémas et inversement, toutes les reterritorialisations ne sont pas menées jusqu'à leur terme. De plus, le degré de contrôle de l'État ou du gouvernement local est extrêmement variable et les politiques de protection de la nature mises en place peuvent donner lieu ou participer à des reterritorialisations autonomes, «nouveau potentiel nomadique» (Deleuze et Guattari, 1980 : 480) d'ailleurs rarement anticipé par les fonctionnaires locaux et par les bailleurs de fonds des aides nationales et internationales.

\section{Méthodologie}

L'étude s'appuie essentiellement sur les résultats des enquêtes effectuées entre 2003 et 2008 dans le cadre du Centre de recherches insulaires

2. Il n'y a pas de définition unique des aires marines protégées. Pour une revue des définitions et de leur usage stratégique dans le cadre des échanges internationaux, voir Salvat, Cazalet et Féral (2007).

3. Gilles Deleuze et Félix Guattari ont créé et utilisé ces notions dans un cadre philosophique et théorique et en donnent, dans la conclusion de Mille Plateaux (1980, 634-636), une définition très claire, utilisable dans le cadre de ce travail : « La fonction de déterritorialisation : D est le mouvement par lequel on quitte le territoire. C'est l'opération de la ligne de fuite. Mais des cas très différents se présentent. La D peut être recouverte par une reterritorialisation qui la compense [...]. N'importe quoi peut faire office de reterritorialisation, c'est-à-dire "valoir pour" le territoire perdu ; on peut en effet se reterritorialiser sur un être, sur un objet, sur un livre, sur un appareil, sur un système... Par exemple, l'appareil d'État est mal dit territorial : il opère en fait une $\mathrm{D}$, mais immédiatement recouverte par des reterritorialisations sur la propriété, le travail et l'argent (il va de soi que la propriété de la terre [et du lagon], publique ou privée, n'est pas territoriale, mais reterritorialisante). » (Le texte mis entre crochets l'est par les auteurs.) 
et Observatoire de l'Environnement (UMR EPHECNRS). Dans l'île de Moorea, une double démarche a été privilégiée. D'une part, une approche qualitative de la perception du concept du PGEM qui a consisté à aborder la question des aires marines protégées dans chaque commune à partir d'un dialogue avec toutes les personnes concernées : autorités communales, pêcheurs, hôteliers, prestataires de services (notamment en ce qui concerne le nourrissage des raies), défenseurs de l'environnement, membres de l'administration en charge du PGEM de Moorea (principalement, les services de l'urbanisme et de l'aménagement, de la pêche et de l'environnement). D'autre part, une approche quantitative de l'utilisation du lagon sur la base des données recueillies auprès des services administratifs ${ }^{4}$. Ces données ont été systématiquement comparées avec celles obtenues par des enquêtes de terrain basées sur des questionnaires et des interviews non directifs. Cette approche a permis de constituer un fonds statistique sur les activités de tourisme et de pêche dans le lagon de Moorea.

Ce travail est organisé en quatre parties. Dans une première partie, nous montrons que le statut traditionnel de l'environnement et celui de la gestion des ressources s'intègrent aussi bien dans une idéologie que dans une structure sociale dotée d'institutions originales. Les représentations et les usages s'inscrivent dans des trajectoires historiques particulières. La seconde montre en quoi ces tissus sociaux et locaux sont déstabilisés par de nouveaux quadrillages administratifs et spatiaux. La continuité terre-mer, jadis en vigueur, est alors ignorée pour donner lieu à de nouvelles pratiques. S'appuyant sur l'exemple de l'île de Moorea, la troisième partie montre concrètement les logiques d'interruption de la continuité terre-mer, à l'occasion de la mise en place d'un plan de gestion de l'espace maritime (PGEM). Ce dernier apparaît ainsi comme le produit des dynamiques historiques antérieures et contribue à fonder un nouveau modèle de gestion des ressources. Compte tenu de ces dynamiques, la quatrième partie analyse le statut ambigu du PGEM aujourd'hui, à la fois outil de gestion souhaité par les populations et outil détourné et recomposé selon des logiques d'acteurs.

\section{Statut traditionnel de l'environnement : représen- tations, hiérarchies et usages}

\section{Cosmogonies et rapports à l'environnement}

Bien qu'il existe dans les langues polynésiennes contemporaines un terme d'origine latine « natura » pour nommer la nature, ceci n'est que récent, les Polynésiens ne disposaient pas de mot particulier (Davies, 1851; Jaussen, 1996). La culture et la nature sont confondues. La nature (c'est-à-dire l'ensemble des entités non humaines) est au cœur des cosmogonies mä'ohi. En effet, les cosmogonies polynésiennes, à la différence de la plupart des mythes occidentaux, intègrent les entités non humaines (coraux, poissons....) dans leurs idéologies. On trouve dans celles-ci trois caractéristiques principales. La première intéresse la fondation de l'univers. Elle résulte d'un processus continuel d'expansion environnementale où les dieux, les demi-dieux et les humains sont eux-mêmes les produits de ce développement cosmique. Ainsi, comme le rappelle B. Rigo :

«Le minéral produit le végétal; le végétal génère l'animal; le divin engrosse le minéral: "Tiki-leseigneur, s'accouplant à une pierre, produisit de la chair rouge" (Métraux, 1980), tandis que Tane [dieu polynésien], en quête d'un élément féminin, copule avec la forêt (Hinewaoriki), avec la montagne (Hinetuparimaunga), avant de fabriquer la femme avec la terre rouge (Hineahuone). » (Rigo, 2004 : 225)

En second lieu, les cosmogonies établissent un principe de continuité verticale descendante et ascendante entre le monde invisible des dieux et des ancêtres et le monde visible des humains et des non-humains (plantes, poissons, récifs....), contrairement aux mythes créateurs européens monothéistes qui instaurent une rupture ontologique entre Dieu et les hommes. En vertu de ce principe de continuité où les dieux et les humains sont généalogiquement liés, chaque famille élargie attachée à un marae disposait de ses propres règles, dont l'institution d'un rāhui (Oliver, 1974 : 770) (voir ci-dessous).

La troisième caractéristique, enfin, est la reconnaissance d'un principe d'interaction permanente entre ces différentes entités sociales, familiales, culturelles ou spatiales. Les cosmogonies permettent ainsi des changements de référence à tel ou tel dieu ou ancêtre, des modifica-

4. Il s'agit notamment: a) des informations relatives à la démographie, aux revenus et aux activités touristiques des populations de Moorea fournies par l'Institut de la statistique de la Polynésie française ; b) de la base de données relative aux pêches lagonaires à Moorea, fourni par le service de la pêche ; c) de différents plans de zonage du plan général d'aménagement (PGA) et du plan de gestion de l'espace maritime (PGEM) fourni par le service de l'urbanisme et de l'aménagement; d) d'informations relatives aux détenteurs de la carte professionnelle de pêcheurs lagonaires à Moorea, fournie par la chambre d'agriculture et de la pêche lagonaire. 
tions des normes dans le contexte d'une interaction permanente entre les entités divines, humaines et non humaines. On est là dans un univers mouvant, fluide et flexible où les cosmogonies apparaissent comme des guides et des fondements pour l'action et la décision. Elles fondent le pluralisme de la structure sociale.

Si l'on passe du monde « idéel » (Godelier, 1984) vers le monde matériel, le marae - temple polynésien à ciel ouvert - revêt une importance fondamentale dans le rapport des Polynésiens à leur environnement.

\section{Marae, hiérarchies sociales et rapports normatifs à l'environnement}

Depuis les travaux de D. Oliver (1974), il a été défini des rôles spécifiques pour les marae au sein de toutes les hiérarchies sociales de la société polynésienne. Mentionnons le marae fêtii qu'ont en propre les membres des familles élargies ou 'ópu et le marae de chefferie qui pouvait à la fois servir de marae familial du chef et de marae de la chefferie. La congrégation des pêcheurs avait son propre marae. Il est probable que le tahu'a tautai - expert de la pêche - contrôlait les territoires de pêche associés aux portions de terre qui appartenaient à sa famille élargie. La logique territoriale polynésienne est profondément topographique. Le marae apparaît comme un surdéterminant de la parenté, du pouvoir politique et religieux et des droits fonciers et marins. On est allié ou parent (fêtii) par rapport à tel ou tel marae.

Quatre hiérarchies sociales principales peuvent être distinguées : les arii, les tahu'a, les raatira et les manahune. La traduction de ces quatre termes est délicate. Le arii était le chef d'un territoire et d'une population qu'il ne contrôlait pas directement, le soin en étant laissé au raatira: chef secondaire puissant qui avait en charge le contrôle du territoire du arii. Parfois nommé chef de terre, le raatira s'occupait des ressources du territoire sous son contrôle, des travaux d'importance entrepris ou des rites agraires selon les époques de l'année. Le grand arii ou arii nui pouvait avoir sous sa coupe plusieurs autres arii moins puissants ainsi que de nombreux raatira dont le nombre variait en fonction de l'allégeance de ces derniers envers le arii. La classe la plus basse de la société était les manahune, qui n'avaient que le contrôle exclusif de leur territoire familial, tout en travaillant pour les raatira et les arii. Chacune de ces trois classes disposait d'un marae familial, à partir duquel les membres des familles élargies dérivaient leurs droits d'usage des terres et de la portion de lagon attachée à ce marae.

Il reste à mentionner le statut de prêtrespécialiste (tahu'a). Le tahu'a est le lien privilégié dans la communication avec les dieux (atua) et les ancêtres. Son rôle est transversal par rapport aux autres classes (Baré, 2002 :177). Si nous suivons le témoignage de James Morisson, quel que soit le rang familial, tout marae fêt tii avait aussi un tahu'a familial ${ }^{5}$. Chaque famille élargie ('oppu) semblait donc former un groupe socio-normatif dans lequel les interactions avec les ancêtres déifiés et les dieux familiaux étaient sans cesse construites et actualisées par toutes les « classes » de la société.

Sur le plan de la gestion foncière et maritime, les droits et les obligations se superposaient en quelque sorte en fonction des hiérarchies sociales formant des sphères légales enchevêtrées. Chacune des classes avait un droit exclusif sur son territoire familial. Mais le arii, comme le raatira et le chef des familles élargies ('ōpū), le matahiapo, disposaient de droits solennels sous la forme des premières prises de pêche, des premières récoltes agraires ou des premières cueillettes, qui leur étaient réservées.

\section{Les dynamiques du rāhui}

Une des modalités concrètes de gestion d'un territoire terrestre et maritime par les Polynésiens réside dans une institution nommée rāhui. Oliver classe la notion de rāhui parmi les concepts sacrés de la cosmogonie polynésienne (Oliver, 1974 : 67). Le rāhui est sans doute une des fonctions les plus importantes d'un chef à la tête d'un groupe de parenté. Il se caractérise par la propriété qu'a le arii de poser des restrictions sur les activités de pêche, de cueillette ou d'agriculture. Les restrictions pouvaient être de différentes natures : activités productives en général ou sur certains produits spécifiques, ou sur la consommation de certains aliments, ou encore sur des activités de construction. Signe d'une continuité à la fois cosmogonique et spatiale, le rāhui était souvent imposé depuis le sommet de la montagne jusqu'à la barrière de récifs, dans un territoire donné sous la coupe d'un chef (arii ou raatira).

Certains observateurs ont défini le rāhui comme étant le système prônant des mesures de conservation et/ou d'interdiction visant à protéger l'environnement, de la part d'administrateurs avisés (souvent les chefs), pour permettre la

5. Ce dernier précise : « chaque chef de famille a le sien, et il y a des offrandes et des prières fréquentes sinon régulières » (Morrison, $1956: 51$ ). 
régénérescence d'espèces ou de productions en danger ou en voie d'extinction. Cela étant, le rāhui mā'ohi est plutôt délibérément imposé pour le bénéfice de ceux qui sont à son initiative. Il peut s'agir de préserver des ressources en préparation d'un événement qui exige une cérémonie touchant toute la communauté, mais aussi, plus implicitement, d'asseoir une suprématie sociale ou politique.

\section{Missionnaires, colonisation et interruption de la continuité terre-mer}

Avec l'arrivée des missionnaires de la London Missionary Society (LMS) en Polynésie, les rapports entre les hommes, l'environnement et le sacré sont profondément transformés. Le arii Pomare II se convertit au protestantisme et bat les « traditionalistes », opposés à la conversion, lors de la fameuse bataille de Fei-Pi en 1815. Peu de temps après, il centralise le pouvoir, s'arroge l'exclusivité du titre de arii, tandis que les autres chefs ne sont plus que des tâvana (chefs) sous sa coupe. C'est également à cette période que Pomare va organiser le monopole du rāhui à son profit en ce qui concerne le commerce du porc (dont la demande est croissante) avec l'Australie.

Par ailleurs, l'ambition des missionnaires n'était pas seulement religieuse, mais aussi économique. Au milieu des années 1860, les missionnaires de la LMS vont favoriser les grandes plantations de cocoteraies dans les plaines littorales et contribuer à la réinterprétation du rāhui de deux manières : d'une part, en en faisant un outil de gestion et d'organisation des tours de récoltes des noix de coco ; d'autre part, en désacralisant le terme: le rāhui ne s'attache plus prioritairement à la préservation d'une ressource considérée comme sacrée, mais est surtout un outil s'appliquant à la gestion des ressources naturelles et cultivées. Les missionnaires apparaissent donc comme les premiers artisans de l'interruption du principe de continuité sur lequel reposaient les cosmogonies polynésiennes.

Comment a réagi cette société face à la colonisation ? Un nouveau modèle territorial de gestion de l'espace se développe. Le principe de continuité entre le territoire marin et terrestre est interrompu par le quadrillage administratif imposé par l'administration coloniale. Entre 1850 et 1884 , l'interruption de la continuité entre les territoires lagonaire et terrestre revêt plusieurs aspects. Les droits d'usage des différents groupes sociaux sont transformés en droits de propriété. Les propriétés terrestres font l'objet d'enregistrements et sont décrites dans des titres de propriété. À Moorea, de grands domaines fonciers sont alors constitués tout au long du $\mathrm{XIX}^{\mathrm{e}}$ et $\mathrm{du} \mathrm{Xx}^{\mathrm{e}}$ siècle. On y cultive le café, le coprah, le coton et la vanille, produits tous destinés à l'exportation. La grande baie d'Opunohu est le siège de plantations de vanille et de cocoteraies. Le littoral de la côte nord-ouest de Moorea (Haapiti) voit émerger de grandes cocoteraies. Une usine de traitement du coprah voit même le jour (Jullien-Para, 2007). Le district d'Afareitu sur la côte, lui, devient le «district caféier» (Robineau, 1984 : 94).

Quant au lagon, il est intégré au domaine public colonial et est régi par les dispositions du code civil depuis 1866 à Tahiti et à Moorea (Calinaud, 1993). Les hiérarchies sociales traditionnelles ainsi que les normes qui avaient cours sur les espaces lagonaires sont désormais ignorées par les autorités en place. Les chefferies anciennes disparaissent pour laisser la place à des conseils de district. Leurs membres, issus des hiérarchies traditionnelles, sont choisis selon leur docilité aux injonctions du pouvoir central (BOEFO, 1897: 363).

En 1945, les cinq archipels de la Polynésie française sont unifiés sous la forme d'une entité administrative unique. La loi-cadre Deferre, en 1956, puis le statut d'autonomie de gestion de la Polynésie, en 1977, vont procurer une autonomie relative à la Polynésie française ${ }^{6}$.

L'arrivée du Centre d'Expérimentation du Pacifique (CEP) en 1964 va considérablement accélérer les processus de transformation de la société polynésienne. Les hauts cadres administratifs et politiques sont souvent des expatriés et, même lorsqu'ils ne le sont pas, peu de références aux principes de gestion traditionnelle des ressources trouvent leur place dans les réglementations et les lois édictées par la Polynésie française ${ }^{7}$.

\section{Logiques de la gestion administrative des ressour- ces aujourd'hui : PGA et PGEM}

La Polynésie française est désormais devenue une collectivité territoriale d'outre-mer. Sur un plan administratif, l'interruption du principe de continuité terre-mer introduit pendant la période coloniale va s'accentuer avec la mise en place des plans généraux d'aménagement (PGA) et des plans de gestion de l'espace maritime

6. Le statut d'autonomie de la Polynésie française sera modifié plusieurs fois : en 1984, 1990, 1996,2004 et 2007.

7. Ce qui n'est pas le cas de la Nouvelle-Calédonie et de Wallis-et-Futuna. 
(PGEM). Le PGA sur l'espace terrestre comme le PGEM sur l'espace maritime, sont des outils administratifs qui visent à définir des zones d'activités permises et interdites (zone urbaine, touristique, activités de pêche autorisées avec ou sans condition, etc.). Ces outils sont accompagnés d'un arsenal légal de sanctions destiné à faciliter leur application.

À Moorea, le PGA est élaboré en 1995 et renouvelé en 2003, tandis que le PGEM de la commune sera mis en place en 2004. Le premier PGA prévoit un certain nombre de règles s'appliquant à des zones délimitées sur le territoire terrestre, en fonction de la nature de l'occupation de l'espace. $\mathrm{Au}$ moins deux caractéristiques peuvent être notées en ce qui concerne l'élaboration du PGA de Moorea au milieu des années 1990.

Il s'agit essentiellement d'un travail administratif ne tenant pas compte des toponymies locales. La délimitation des zones est théorique et ne coïncide pas avec le relief insulaire. Ce document n'a pas été réalisé en Polynésie française mais par un cabinet spécialisé localisé à Paris.
Les hauts lieux de la culture polynésienne qui parsèment l'île de Moorea n'ont pas fait l'objet d'un relevé ou d'un inventaire détaillé. En 1995, les zones dédiées aux sites archéologiques ne représentaient que 19 hectares (contre 156 hectares dans le PGA de 2003), alors même que les travaux archéologiques ont dressé des inventaires circonstanciés des hauts lieux culturels et politiques anciens à Moorea (notamment, Green et al., 1968 ; Conte, 1999).

Le PGA renouvelé en 2003 est mieux élaboré, plus proche des réalités géographiques, et tient compte des espaces culturels, anciens et nouveaux. Il respecte les nouvelles fonctions urbaines de l'île (zone d'habitation pavillonnaire) dans sa proximité avec la capitale de Tahiti, Papeete. Dès avant les années 1960, l'île de Moorea a, en effet, acquis la fonction de zone péri-urbaine de Papeete. En quarante-cinq ans (de 1962 à 2007), la population de Moorea augmente de près de $300 \%$, passant de 4147 à 16490 personnes (voir tableau 1). Le tableau 2 illustre l'évolution des grandes tendances de la répartition de l'espace communal entre 1995 et 2007.

\begin{tabular}{|l|r|r|r|l|l|l|l|l|}
\hline \multicolumn{9}{|c|}{ Population recensée } \\
\hline $\begin{array}{l}\text { Moorea- } \\
\text { Maiao }\end{array}$ & 1962 & 1971 & 1983 & 1988 & 1996 & 2002 & 2007 \\
\hline & 4147 & 4840 & 7249 & 9032 & 11965 & 14471 & 16490 \\
Afareaitu & & 1163 & 1565 & 1864 & 2447 & 2912 & 3249 \\
Haapiti & & 1034 & 1572 & 2010 & 2885 & 3463 & 4045 \\
Paopao & & 1335 & 1914 & 2413 & 3085 & 3852 & 4244 \\
Papetoai & & 699 & 998 & 1328 & 1740 & 1879 & 2196 \\
Teavaro & & 609 & 1010 & 1186 & 1525 & 2057 & 2457 \\
Maiao & & & 190 & 231 & 283 & 308 & 299 \\
\hline
\end{tabular}

TABleau 1. - Évolution démographique à Moorea

Source : ISPF (2008)

\begin{tabular}{|l|c|c|c|c|}
\hline $\begin{array}{l}\text { Les espaces d'habitation } \\
\text { 1995-2007 }\end{array}$ & Village & $\begin{array}{c}\text { Habitat rural } \\
\text { diffus }\end{array}$ & $\begin{array}{c}\text { Habitat } \\
\text { pavillonnaire }\end{array}$ & $\begin{array}{c}\text { Habitat propriétaire } \\
\text { proche de zones } \\
\text { agricoles }\end{array}$ \\
\hline 1995 (zonage en ha) & 408 & 1500 & 0 & 274 \\
\hline $\mathbf{2 0 0 7}$ (zonage en ha) & 415 & 1374 & 100 & 652 \\
\hline Évolution en \% & $+1,7 \%$ & $-8,4 \%$ & Ind. & $+137 \%$ \\
\hline
\end{tabular}

Source : service de l'Urbanisme (2008)

TABLEAU 2. - Évolution du découpage de l'espace communal à Moorea

En dépit d'une meilleure prise en compte de l'occupation de l'espace sur l'île, les dispositifs administratifs demeurent, dans leurs principes, dans la même logique de rupture culturelle entre la terre et le lagon. En effet, le PGA ne s'intéresse qu'à la partie cadastrée du milieu terrestre et ignore les parties émergées non cadastrées. À ce titre, il est particulièrement intéressant de relever 


\begin{tabular}{|c|c|c|}
\hline $\begin{array}{l}\text { Aire } \\
\text { marine } \\
\text { protégée } \\
\text { (AMP) }\end{array}$ & $\begin{array}{l}\text { Zonage du plan Gestion de l'espace } \\
\text { maritime (PGEM) }\end{array}$ & $\begin{array}{l}\text { Zonage du plan général } \\
\text { d'Aménagement (PGA) }\end{array}$ \\
\hline Tiahura & Récréative et scientifique. Nord Haapiti Pk 26 & $\begin{array}{l}\text { UTC : zone touristique } \\
\text { UC : zone hors agglomération }\end{array}$ \\
\hline Tetaino & Interdite à la pêche. Nord ouest Haapiti Pk 28 & UC : zone hors agglomération \\
\hline Taotaha & $\begin{array}{l}\text { Vocation halieutique. Pêche interdite. } \\
\text { Nord ouest Haapiti Pk } 30\end{array}$ & UC : zone hors agglomération \\
\hline Maatea & $\begin{array}{l}\text { Vocation halieutique. Pêche interdite. } \\
\text { Afareitu, Pk } 14\end{array}$ & UB : zone urbaine \\
\hline Ahi & Vocation halieutique. Afareitu, $\mathrm{Pk} 8$ & $\begin{array}{l}\text { NRh : zone de forte houle } \\
\text { NDd : littoral }\end{array}$ \\
\hline Nuarei & Vocation récréative. Est de l'île, Teavaro & UTC : zone touristique \\
\hline Aroa & $\begin{array}{l}\text { Vocation halieutique. Nord-est, Paopao, } \\
\text { Pk } 4\end{array}$ & $\begin{array}{l}\text { UT : zone touristique (en limite) } \\
\text { UC : zone hors agglomération }\end{array}$ \\
\hline Pihaena & Vocation halieutique. Nord baie Paopao & $\begin{array}{l}\text { UC : zone hors agglomération } \\
\text { NDd : Littoral } \\
\text { NRh : zone de forte houle }\end{array}$ \\
\hline
\end{tabular}

Source : service de l'Urbanisme (2008)

TABLEAU 3. - Localisation des aires marines protégées par rapport au zonage du plan général d'aménagement à Moorea

les logiques à l'œuvre en analysant la localisation des huit aires maritimes protégées par rapport aux zonages du PGA en vigueur à Moorea (voir tableau 3).

Il semble que la logique environnementale soit la seule qui ait été retenue pour le choix des aires marines protégées. Mais aucun des documents administratifs consultés ou des relevés de décision du comité permanent du PGEM n'en fait état.

L'espace occupé par les villages passe entre 1995 et 2003 , de 408 à 415 hectares $(+1,7 \%)$ (voir tableau 2). Aucun accès à la mer n'est prévu pour les espaces villageois (zone urbaine UB) ${ }^{8}$. Tout au plus, le nouveau PGA relève-t-il des zones littorales inconstructibles (zones de forte houle NRh et zone du littoral NDd) en raison des fortes houles saisonnières, permettant des aménagements touristiques, culturels ou archéologiques. Ces dernières zones n'ont cependant pas été délimitées du fait de la présence des espaces villageois et ne permettent pas aux pêcheurs lagonaires d'y déposer leurs pirogues.

L'habitat pavillonnaire s'est considérablement développé depuis le début des années 1980 et se caractérise par un étalement des habitations le long du littoral. La répartition de ce type d'habitat ne permet désormais plus l'accès traditionnel au lagon. La zone d'extension de l'habitat pavillonnaire, absente du PGA de 1995, occupe en 2003 près de 100 hectares de surface (voir tableau 2). Au total, l'espace pavillonnaire représente désormais $20 \%$ du territoire destiné à l'habitation.

Les grandes infrastructures hôtelières sont essentiellement localisées sur la côte nord-nordouest de l'île de Moorea, là où les plages de sable blanc sont les plus belles. La zone accordée aux activités touristiques par l'administration passe de 171 à 279 hectares entre 1995 et 2003 (+ $63 \%$ ). Les seules correspondances entre PGEM et PGA concernent les zones terrestres à vocation touristique (UT) et les AMP dites récréatives (Tiahura et Nuarei). Beaucoup de zones dites UT sont localisées dans - ou en bordure - des AMP interdites à la pêche. Les interdictions qui s'appliquent aux pêcheurs ne concernent pas les acteurs des activités récréatives.

Enfin, comme dans le PGA de 1995, les toponymies traditionnelles terrestres et lagonaires sont ignorées.

La forte pression démographique s'exerçant depuis la moitié $\mathrm{du} \mathrm{xx}^{\mathrm{e}}$ siècle, la rupture de la continuité terre-mer provoquée par le quadrillage administratif de chacun des espaces, la constitution de grands domaines fonciers, l'urbanisation croissante de l'île et l'appropriation des plus beaux sites littoraux par l'industrie touristique ont participé, à Moorea, à l'enclavement des populations locales au sein des villages et à la réduction de leur accès au lagon. Compte

8. Nous n'avons trouvé aucune correspondance entre les zones urbaines (UB) et les zones du littoral (NDd) hormis le port de Vaiare où circulent de très gros bateaux. 
tenu de ce qui a été indiqué, il convient maintenant d'examiner quels ont été les effets directs et induits de la mise en place récente du PGEM dans le lagon de Moorea.

\section{Les effets territoriaux du PGEM}

Le PGEM de Moorea procède d'une « reterritorialisation » au sens de Deleuze et Guattari (1980), à la fois parce que sa mise en place s'inspire d'une conception importée, celle de la préservation de l'environnement, mais aussi parce qu'elle contribue à des recompositions territoriales que personne n'avait sans doute prévues ni envisagées.

Suivant les distinctions méthodologiques proposées par Di Méo (1998), trois types de recomposition territoriale peuvent être distingués, les types fondés sur a) la représentation spatiale des composantes du territoire; b) l'utilisation du territoire selon la règle ; c) l'utilisation du territoire selon les usages.

\section{Le PGEM : un quadrillage administratif des compo- santes du territoire}

La mise en place du PGEM, entamée au début des années 1990, aboutit en 2004. Cette mise en œuvre procède de la même logique de déterritorialisation que celle qui a débuté avec l'institution des droits de propriété et des espaces privés/publics. Elle apparaît ainsi comme l'aboutissement de cette logique qui s'applique désormais au territoire lagonaire et constitue un des dispositifs qui participent à la rupture de la continuité terre-mer dans la conception polynésienne.

Pour illustrer ce propos, il est intéressant d'analyser le préambule du règlement du PGEM de l'île de Moorea :

"Moorea [...] né d'un point chaud il y a environ 2 millions d'années [...]. Jusqu'à la seconde guerre mondiale, l'économie de l'île était basée sur le coprah, la vanille et le café. [...] Les écosystèmes coralliens sont particulièrement riches en espèces et sont souvent considérés comme de véritables oasis. De ce fait, de nombreuses activités économiques s'y exercent. Afin d'assurer un développement durable aux populations qui dépendent de cet environnement, il est indispensable de favoriser la gestion de cet espace complexe, diversifié, productif mais également fragile. Cette gestion doit intégrer les souhaits et la dynamique des populations littorales. C'est le rôle du plan de gestion de l'espace maritime (PGEM) dont la procédure est définie par le code de l'aménagement de Polynésie française (articles D.133-1 à D.133-10). [...] »

Ce préambule joue en quelque sorte la fonction d'un nouveau mythe sur lequel se fonde le règlement du PGEM de l'île. Notons trois caractéristiques importantes. D'une part, pour mieux fonder le projet, l'histoire s'inscrit dans un lointain géologique qui débute il y a deux millions d'années. D'autre part, l'histoire humaine débute à la Deuxième Guerre mondiale. L'impasse est faite sur les deux mille dernières années au cours desquelles des conceptions très différentes de l'espace et du territoire avaient cours et soutenaient des institutions originales telles que le rāhui. Le préambule présente des activités considérées comme éternelles ("Jusqu'à la Seconde Guerre mondiale »), alors qu'il ne s'agit que des quatre-vingts dernières années qui ont précédé la période indiquée. Enfin, les présupposés avancés sont essentiellement d'ordre environnemental.

En même temps, cette représentation administrative du territoire des AMP ne reste pas univoque ni unilatérale. Elle entre en compétition avec d'autres représentations. En effet, si l'on passe d'une échelle territoriale à une échelle locale et d'un contexte administratif à une situation de forum délibératif (le comité permanent du PGEM de Moorea), les représentants au titre de l'environnement et de la culture au sein du comité de gestion permanent du PGEM ont été désignés par le maire et les autres membres du comité pour réfléchir et choisir à la fois un logo et une maxime qui symboliseraient le PGEM. Leurs propositions retenues sont devenues les symboles publiquement affichés du PGEM de Moorea. À ce titre, il est intéressant de mentionner les raisons avancées pour le choix du logo (une pieuvre $f e^{\prime} e$ ) et de la maxime (le lagon pour les générations futures Te tairoto no te u'i tau).

"Autrefois, dans la croyance du peuple polynésien, Ta'aroa est dieu; il est le ciel, la terre, l'océan, le vent, la pluie, la nature de la terre. [...] En guise de remerciement, les Polynésiens offraient à leur dieu, le Oho Matamua, c'est-à-dire... la première cueillette, l'offrande des premiers fruits [...]. Pour que la paix règne sur terre, Taaroa envoya un gardien sur terre, preuve de son existence. Le symbole visible fut le fe'e (pieuvre), symbolisant taaroa lui-même. [...] on l'appelait taumata-fe'e-fa'atupu-hau ou tumu-ra'i-fenua [...]. Plusieurs générations vivaient en harmonie... quand, un jour, des étrangers (taata honu) arrivèrent à Aimeho-Nui [ancien nom de Moorea]. Les habitants les accueillirent à bras ouvert, sauf le gardien, car ces étrangers amenèrent également leurs idéaux différents de ceux du peuple d'Aimeho. Au fur à et mesure que le temps passait, la discorde régna entre eux. Malgré les mises en garde de Tau-mata-fe'e-fa'atupu-hau, les natifs renonçaient à l'écouter. Alors, il annonça: "Je retourne vers mon père car je n'ai plus à être là. Mais je ne reviendrai que lorsque règneront la paix, le bien et l'unité". À ce moment-là, il émît l'encre sur l'île qui se déversa dans le lagon de Vaihere et de Tahiamanu. 
Tous les êtres vivants marins furent empoisonnés. Le versant ouest du mont Rotui, à Vaihere, témoigne de ce fait. $\gg{ }^{9}$.

On remarque que les propos avancés ne sont pas dénués d'ambiguité et jouent une fonction explicative des conflits d'usage sur le lagon.

La structure du texte est particulièrement intéressante car elle reprend les éléments-clés de cosmogonies polynésiennes identifiées dans la première partie de cet article. La vie est perçue comme une croissance continuelle et continue (Taaroa est ciel, terre, océan, pluie, etc.). La communication entre entités visibles et invisibles est constante (Taaroa envoie un gardien : la pieuvre fee) et implique des interactions (Taaroa, déçu par le comportement de ses protégés, retire son gardien).

Si nous pouvons noter le caractère syncrétique du texte autant inspiré par les croyances traditionnelles que par la Bible, les explications fournies pour le choix du logo (la pieuvre) et de la maxime visent à interpréter en termes culturels : a) l'importance de la prise en compte de la continuité terre-mer pour la gestion durable des ressources; b) l'interruption de cette continuité associée à la venue d'étrangers (taata honu); c) en dépit des mises en garde, l'abandon - temporaire - de Taaora, dieu polynésien, du fait de l'abandon par les insulaires de leurs manières de faire ; d) les conflits qui s'ensuivent depuis cette époque.

Si l'on passe maintenant du territoire des représentations à celui des règles, il est intéressant d'analyser les logiques à l'œuvre dans le champ social.

\section{Le territoire de la règle}

La présentation du règlement du PGEM de Moorea indique :

« [...] Le plan de gestion de l'espace maritime [...] est cohérent avec la politique territoriale mais spécifique à l'île concernée. Il se veut un modèle de gestion intégrée, outil nécessaire au développement durable. » (JOPF, $2004: 420$ )

La mise en place d'un PGEM se matérialise par un quadrillage de l'espace lagonaire. L'ensemble du lagon fait l'objet d'une réglementation iden- tifiant huit aires marines réparties autour de l'île dotées d'une protection renforcée (figure 1). Chaque AMP fait l'objet d'une spécificité. Toutes les activités sont désormais spatialement limitées (pêche, loisir, activités nautiques...) et certaines sont circonscrites à un lieu déterminé (baleinedans les baies -, nourrissage des raies et des requins, pêche au ature, chinchard). Le règlement du PGEM prévoit trois chartes : une pour la pêche aux ature, une pour le nourrissage des raies et une pour celui des requins. La première sera en vigueur de 2005 à 2006, puis ne sera plus renouvelée. Les deux dernières ne sont pas encore entrées en application. Le texte du PGEM est complexe et les interdictions de pêche (au niveau des techniques utilisées ou touchant à leurs fréquences...) sont spécifiques à chaque $\mathrm{AMP}^{10}$.

Tenant compte de la nouvelle réglementation en matière d'AMP, le territoire de pêche a été réduit d'au moins quatre manières :

- une réduction des espaces lagonaires ouverts à la pêche. Les huit AMP qui quadrillent le lagon de Moorea limitent tous les accès des pêcheurs (par interdiction absolue ou par limitation des techniques utilisées). Elles représentent au total presque $20 \%$ de la superficie totale du lagon de Moorea ;

- pour les deux grandes zones de pêche localisées au nord de l'île, qualifiées de « zone spéciale de pêche réglementée » (articles 9 à 14 et 68 du règlement du PGEM), la taille des prises, les techniques et les espèces font également l'objet d'une réglementation qui en limite théoriquement les prises ;

- en ce qui concerne les grandes pêches collectives aux ature-chinchard -, le nombre de pêches pratiquées par pêcheur est également limité à une pêche annuelle. Compte tenu des moyens économiques déployés pour cette pêche collective et traditionnelle (Ottino, 1965 ; Blanchet, 1990), une telle limitation pourrait être de nature à en entraîner sa disparition ;

- toutes ces interdictions ne font l'objet d'aucune mesure compensatrice pour dédommager les pêcheurs (aide à la reconversion, aide aux modifications des techniques de pêche, etc.). De plus, la cohérence entre le PGEM et la politique territoriale, mentionnée en préambule, ne semble pas évidente. En effet, le paradoxe réside dans le fait que le PGEM, figurant dans le code de l'aménagement, limite les efforts de pêche alors que, dans le même temps, la détention d'une carte professionnelle de pêche permet d'obtenir des réductions (outils, carburant....) ou des subventions (" machines à glace,

9. Le document bilingue n'est pas daté. Il a été écrit par Christa Teihotu (représentante au comité de gestion au titre de l'Environnement) et Maurice Rurua (Culture). L'écriture a été respectée.

10. À titre d'exemple, l'AMP de Maatea au sud de l'île est régie par diverses règles : - Pêche aux ature interdite (art 62); - Dispositions communes (art 48) + Interdits sauf pêche à la ligne et à la traîne (art. 63) ; du récif au chenal : - Dispositions communes (art 48) + Interdits sauf pêche au filet d'une longueur de $50 \mathrm{~m}$ maxi et maille mini $50 \mathrm{~mm}+$ pêche fusil de jour (art. $63)$. 


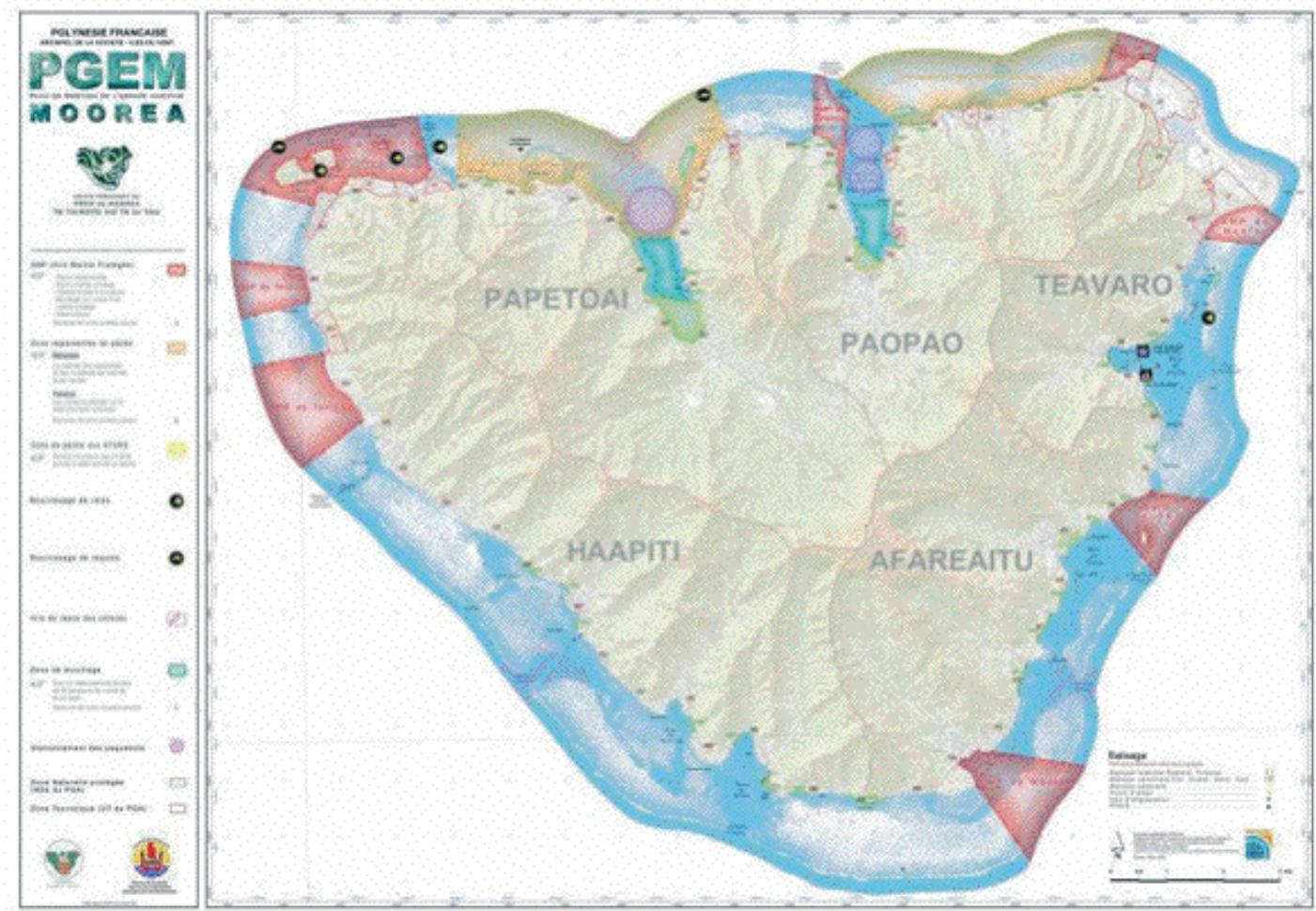

Figure 1. - Carte du PGEM de Moorea

cessions de formation ${ }^{11}{ }^{1}$ ) et encourage l'intensification des efforts de pêche dans le lagon ${ }^{12}$. Cette incohérence se traduit également par la faiblesse du contrôle du territoire marin de la part des autorités locales et territoriales. En effet, si des règles précises réglementent des espaces marins et/ou la collecte de ressources spécifiques, la Polynésie française a déployé peu de moyens pour que les infractions soient sanctionnées en cas de délit. Les difficultés tiennent tout autant à la difficulté du constat matériel de ces infractions qu'à l'absence de poursuites et de sanctions. Entre 2005 et 2006, seules 40 infractions sur le lagon ont fait l'objet d'un procès-verbal et transmises au procureur. Selon notre enquête, aucune n'a fait l'objet de poursuite et de sanction. Qu'il s'agisse des infractions relatives aux pêches interdites, au mouillage, à la vitesse de circulation dans le lagon, à l'occupation du domaine public maritime, ou au non respect des règles du PGEM, les contrôles et les sanctions sont trop rares pour être réellement dissuasives ;

- enfin, le comité permanent du PGEM qui veille au respect de celui-ci n'a qu'un rôle consultatif. Il ne peut en aucun cas se substituer aux autorités territoriales pour imposer des amendes.
Dans le contexte de Moorea, où les zones terrestres dédiées aux activités touristiques ont fortement augmenté entre 1995 et $2003(+28 \%)$, il est particulièrement instructif de relever les logiques à l'œuvre dans l'élaboration de ces dispositifs publics, spécialement dans le cas des zonages touristiques. Ainsi, les mesures de gestion administrative du territoire (terrestre et marin) introduisent une forte ségrégation spatiale : ce qui est interdit à la pêche est autorisé aux activités récréatives. Le PGA ne prévoit pas d'accès au littoral pour les pêcheurs en face des villages (pas de zones NDd face aux villages) mais privilégient les zones touristiques (UT et UTC) dans les $\mathrm{AMP}^{13}$ ou face à elles, ou à proximité (voir tableau 4).

\section{Le territoire des usages}

Un découpage de l'espace lagonaire calqué sur le développement touristique

Si les espaces lagonaires et terrestres dédiés aux activités touristiques sont importants et en

11. Lettre du ministre de la pêche, avril 2006. Te Vea Tautai (littéralement : «journal de la pêche ») 18.

12. Le dossier relatif à la pêche lagonaire du Te Vea Tautai 18, p. 3, indique : « Cette carte confère à son porteur un caractère professionnel et lui donne droit de bénéficier de certaines aides pour l'acquisition de certains équipements de base ainsi que pour une embarcation et/ou un moteur. [...]».

13. Les hôtels bénéficient de la proximité, voire sont situés dans, des AMP dites récréatives ou dans des zones de pêches réglementées ou totalement interdites à la pêche. 


\begin{tabular}{|l|l|l|}
\hline \multicolumn{1}{|c|}{$\begin{array}{c}\text { Localisation des zonages } \\
\text { touristiques (UT OU UTC) }\end{array}$} & \multicolumn{1}{|c|}{$\begin{array}{c}\text { Nature de l'AMP ou type de zone } \\
\text { lagonaire }\end{array}$} & \multicolumn{1}{c|}{ Hôtels } \\
\hline Tiahura Pk 26 & $\begin{array}{l}\text { AMP récréative et scientifique. } \\
\text { Nord Haapiti }\end{array}$ & $\begin{array}{l}\text { Club Méditerranée } \\
\text { (fermé) }\end{array}$ \\
\hline $\begin{array}{l}\text { Entre les AMP Tetaino et Taotaha } \\
\text { Pk 28-30 }\end{array}$ & Interdite à la pêche. Nord ouest Haapiti & $\begin{array}{l}\text { Hôtel Moorea Village } \\
\text { (fermé) }\end{array}$ \\
\hline $\begin{array}{l}\text { Temae } \\
\text { AMP de Nuarei }\end{array}$ & $\begin{array}{l}\text { Vocation récréative. Est de l’île, Teavaro } \\
\text { Hors AMP, nourrissage de raie autorisé }\end{array}$ & Hôtel Sofitel \\
\hline En bordure de l'AMP Aroa Pk 4 & Vocation halieutique. Nord-est, Paopao & Moorea Pearl Beach \\
\hline Maharepa & $\begin{array}{l}\text { Zone de pêche réglementée } \\
\text { Nourrissage de requin autorisé }\end{array}$ & Hôtel Sheraton \\
\hline Paopao PK7 & Zone mouillage paquebot & Hôtel Bali Hai Club \\
\hline Paopao, PK 8,7 & Zone de mouillage réglementée & Hôtel InterContinental \\
\hline Pointe de Vaipahu Pk14 & En limite de zone de pêche réglementée & \\
\hline Vaihere Pk 15. Opunohu & Zone de pêche réglementée & $\begin{array}{l}\text { AMP récréative Tiahura. Nourrissage de } \\
\text { raie autorisée }\end{array}$ \\
\hline Tepee Pk 24,5. AMP de Tiahura & & \\
\hline
\end{tabular}

Source : service de l'Urbanisme (2008)

TABleaU 4. - Emplacement des hôtels et des AMP

augmentation, favorisés en cela par les dispositions administratives et réglementaires, cela ne suffit pas à caractériser ces espaces de « territoire touristique ». Sur ce dernier point, il faut en effet analyser de quelles manières ces espaces particuliers sont socialisés (Raffestin, 1986 ; Di Méo, 1998).

Le « territoire hôtelier » se caractérise d'abord par un contrôle de l'espace. La présence des hôtels est évidente, que ce soit depuis le littoral, avec des entrées imposantes, ou dans le lagon avec les bungalows sur pilotis. Toutes les structures font appel à des sociétés de gardiennage pour contrôler leurs accès.

Dans le cadre du comité permanent du PGEM, les hôteliers disposent d'un représentant qui est le directeur de l'un des quatre hôtels classés de Moorea. Si ces quatre établissements de classe internationale sont en situation de concurrence classique les uns vis-à-vis des autres, ils parlent d'une seule voix pour défendre un territoire et des intérêts communs : la modification de leur surface maritime, la construction d'un ponton, etc. L'analyse des procès-verbaux du comité permanent du PGEM montre que les dossiers relatifs aux demandes de concession du domaine public maritime de la part des acteurs touristiques occupent, en général, une grande partie des réunions de ce comité. Dans tous les cas, force est de constater que les hôteliers ont une bonne connaissance du texte du PGEM ; ils s'efforcent de l'appliquer et de le respecter.

Enfin, l'appropriation de l'espace par les activités récréatives se traduit par l'importance économique des activités développées, que ce soit par les hôteliers directement ou par des prestataires lagonaires indépendants. À titre d'illustration, les prestations touristiques liées au nourrissage des raies pastenagues - en liberté dans le lagon de Moorea - montrent la nature des transformations socio-économiques dans le lagon de Moorea. En effet, des enquêtes réalisées en 2005 selon deux approches différentes, convergent en ce qui concerne l'importance économique de cette activité, créée dix ans auparavant (tableau 5). Le chiffre d'affaires annuel directement lié aux prestations de nourrissage des raies à Moorea oscille dans une fourchette allant de 490000 à $572000 €$. Depuis la mise en place du PGEM en 2004, le zonage des activités a favorisé la diminution du nombre de prestataires, l'augmentation de la taille moyenne de chacun et la réduction du nombre de sites visités - passant de 4 à 2 .

La charte de nourrissage des raies prévue par le règlement du PGEM n'est pas encore entrée en application ${ }^{14}$. Ce vide réglementaire et l'absence d'autorégulation des prestataires ont des effets environnementaux importants. Le nourrissage des raies a, en effet, entraîné une concentration des requins à pointe noire dans cette partie de 


\begin{tabular}{|l|l|}
\hline Estimation 1 & Estimation $\mathbf{2}$ \\
\hline $\begin{array}{l}\text { Capacité journalière des opérateurs de } \\
\text { nourrissage des raies : 400 touristes/jour }\end{array}$ & 136.651 touristes venus à Moorea (ISPF) \\
\hline $\begin{array}{l}\text { Taux de fréquentation moyen annuel (tous } \\
\text { opérateurs confondus) : 40\%, soit } \mathbf{5 8} \mathbf{4 0 0} \text { en } \mathbf{1} \text { an }\end{array}$ & $\begin{array}{l}50 \% \text { participent au nourrissage des raies, soit } \mathbf{6 8 ~ 3 0 0} \\
\text { en } \mathbf{1} \text { an }\end{array}$ \\
\hline Approx. 160 touristes/jour & Approx. $\mathbf{1 8 7}$ touristes/jour \\
\hline
\end{tabular}

Une première approche globale se base sur les données chiffrées des dix prestataires du nourrissage des raies. Leur capacité journalière totale régulière (hors prestations spéciales) en 2005 était de 400 personnes par jour. Le taux de fréquentation est en moyenne de $40 \%$, soit 160 clients par jour. Une deuxième approche est fondée sur les statistiques officielles (ministère du Tourisme et Institut de la Statistique de Polynésie française, ISPF). Sachant que 136651 touristes sont venus à Moorea en 2005 (source ISPF) et considérant que, d'après les observations et enquêtes (Charles, 2005), $50 \%$ de ces touristes participent à une activité de découverte du lagon, on évalue à 68326 le nombre de visiteurs allant à la rencontre des raies, soit une moyenne de 187 personnes par jour (estimation). Ces deux estimations, assez proches, concordent entre elles et avec les informations recueillies sur le terrain : une fréquentation quotidienne moyenne évaluée entre 160 et 187 touristes par jour.

Le prix moyen d'une sortie « découverte des raies» était de 4000 francs CFP en 2005 (34€ environ). La « valeur » liée à la présence des raies est évaluée à $25 \%$ de la prestation, le reste étant constitué de la découverte du lagon et du motu, du repas éventuellement. 1000 francs CFP (soit 8,39€) par visiteur, c'est, théoriquement, le revenu moyen produit par l'activité additionnelle liée à la visite des raies, par touriste. Le chiffre d'affaires annuel en 2005 directement lié aux prestations de nourrissage des raies à Moorea est évalué entre 58,4 millions de francs CFP et 68,3 millions de francs CFP (490 000 et $572000 €$ ). C'est faible (2,3 à 2,7 \%) à l'échelle des recettes d'excursions touristiques (terrestres et lagonaires) réalisées en Polynésie française pendant la même année (2,5 milliards de francs CFP) (ISPF $\mathrm{n}^{\circ}$ 8/2006) (plus de 21 millions $€$ ).

TABleau 5. - Deux évaluations du chiffre d'affaires généré par la découverte des raies pastenagues à Moorea (2005)

lagon, ceci de manière très marquée depuis 2007. Or, l'interdiction du nourrissage des requins dans le lagon depuis 2004 constitue une règle stricte, édictée notamment à la demande des pêcheurs. Maintenant, à chaque nourrissage, entre cinq et quinze requins à pointe noire circulent autour des touristes présents pour la prestation. Si les travaux sur l'impact du nourrissage des raies, leurs déplacements et leur biologie n'aboutissent pas aujourd'hui à des résultats alarmants (Gaspar, 2008), l'absence de suivi de l'attraction des requins et de l'effet de leur concentration autour des zones de nourrissage des raies peut inquiéter.

La corporation des pêcheurs traditionnels au ban de l'espace lagonaire

S'il est difficile de classer les pêcheurs en catégories (Galzin et al., 1989 ; Yonger, 2002 ; Vieux, 2002), la pression exercée sur le lagon par les pêcheurs professionnels ou occasionnels est de plus en plus forte. Selon les sources, la pêche lagonaire de subsistance et commerciale varie entre 400 et 1000 tonnes de prélèvement de la ressource par an, toutes espèces confondues, à
Moorea (Service de la pêche, 2008; Yonger, 2002 ; Mouillot, comm. pers. ${ }^{15}$ ).

Depuis 2004, la réduction des territoires de pêche ne semble pas s'accompagner d'une diminution des prises. Les pêcheurs interrogés admettent en général ne pas respecter les prescriptions du règlement du PGEM. Le sentiment de dépossession d'un territoire au profit des acteurs de la filière touristique est renforcé par le développement anarchique de certaines prestations touristiques.

À ce propos, discutant de la territorialité des AMP, David et Thomassin (2007) parlent de « territorialité identitaire » :

«[...] en Océanie, le regain de territorialité identitaire naît de la rencontre d'une stratégie d'acteur initiée à un niveau supra local (national en l'occurrence) avec un milieu local peu réceptif. »

En ce qui concerne Moorea, il nous semble cependant que la territorialité identitaire est multiforme et plurifactorielle, accentuée par une double dépossession de l'espace insulaire :

D'une part, en raison de l'extension de l'espace pavillonnaire (près de 100 hectares entre 1993 et 2005) et de la réduction des accès au littoral, beaucoup de pêcheurs sont aujourd'hui

15. David Mouillot (2007, communication personnelle) estime le prélèvement de la ressource lagonaire à environ 600700 tonnes par an à Moorea (soit $42 \mathrm{~kg}$ par habitant). Notre propos n'est pas de comparer cette valeur en fonction des sources, mais une évolution des prises ou de la ressource halieutique qui, si c'était le cas, devrait utiliser les mêmes méthodes de travail sur une échelle de temps relativement longue. Nous souhaitons simplement souligner, qu'indépendamment des méthodes utilisées, le niveau de prélèvement de la ressource demeure élevé, avant et après l'application du PGEM. 
confinés dans les espaces villageois ou dans les vallées.

D'autre part, en raison de la nouvelle fonction péri-urbaine de l'île de Moorea proche de la capitale Papeete, les terrains et les domaines ont acquis une valeur très élevée (près de $100 € 1 \mathrm{l}$ mètre carré), si bien que nombreux sont ceux qui n'ont plus accès aux territoires de cueillette jadis accessibles.

\section{Conclusion}

Le territoire lagonaire est à la fois devenu un enjeu de représentation, de régulation et, sur le plan des pratiques, un enjeu conflictuel évolutif qui lie des intérêts opposés (acteurs de la pêche et acteurs d'activités récréatives). Certains de ces enjeux sont multiformes. Ils naissent sur l'espace terrestre pour se prolonger dans le lagon et viceversa. Dans ce contexte, les dispositifs administratifs ne sont pas neutres et privilégient les intérêts touristiques par rapport à ceux des pêcheurs.

Les logiques à l'œuvre dans le champ social ont engendré des effets paradoxaux. La progression touristique, antérieure au PGEM, semble s'inscrire dans une tendance qui se confirme à Moorea, l'île-sœur, qui reçoit plus de la moitié des touristes venant en Polynésie française. Dans le même temps, une grande partie des pêcheurs, sous-valorisée et en manque de reconnaissance, n'adhère pas fondamentalement au PGEM et reconnaît l'enfreindre régulièrement. Dans ces perspectives, le développement touristique, favorisé de longue date au nom du développement, pourra-t-il, seul, garantir la pérennité des ressources (voir aussi Ghasarian et al., 2004) ?

Le rôle et la fonction du PGEM sont ambigus. Plutôt que de redistribuer les cartes, il semble renforcer les tendances contrastées au plan socio-économiques que nous venons d'évoquer, à savoir le poids du secteur touristique soutenu par l'administration. Ainsi, le PGEM apparaît également comme une plate-forme pour des stratégies d'acteurs où tout le monde joue une partition musicale sans harmonie !

Une question à laquelle nous ne pouvons répondre est de savoir si, in fine, l'absence du PGEM aurait été plus favorable à la situation des pêcheurs. Car le PGEM s'impose désormais comme une référence incontournable, que l'on accepte, détourne ou conteste. Contrairement aux pêcheurs, les hôteliers, en particulier, ne peuvent se permettre d'enfreindre les règles du PGEM sans se mettre hors jeu, ce qui est impossible compte tenu de l'importance des investissements réalisés et des contraintes réglementaires qui pèsent sur leurs activités. À cela s'ajoute la position des riverains. Ils représentent la très grande majorité des usagers du littoral et ne sont nullement représentés au sein du comité de gestion du PGEM. L'opposition entre activités récréatives touristiques et activités de pêche est factice. Elle ne tient pas compte du fait que les pêcheurs ont aussi des usages récréatifs du lagon et que la population de Moorea, comme les pensions de famille plus modestes, se plaignent de la rareté des accès à des plages publiques pour des activités récréatives.

En terme territorial, le PGEM a induit des effets contradictoires, fragmentés et complexes, selon les acteurs concernés. Pour les hôteliers, le développement de leur territoire marin passe par la validation, voire l'extension des possibilités de développement que leur accordent leurs concessions maritimes, et cela jusqu'à l'idée de la superposition d'une AMP exclusivement hôtelière. Pour les pêcheurs, le maintien de leur territoire est difficile. Leur stratégie passe à la fois par le non-respect des règles et par leur influence au sein du comité de gestion du PGEM, dans lequel ils peuvent influencer les obtentions d'extension de concessions maritimes.

Enfin, les riverains sont absents des débats au sein du PGEM. Ils n'ont aucune influence malgré leur place grandissante à Moorea depuis plusieurs décennies.

Le PGEM s'impose désormais comme une référence incontournable, qu'on l'accepte, qu'on le détourne ou le conteste. Au-delà des oppositions, le PGEM apparaît comme un outil indispensable de gestion durable des ressources et des territoires malgré l'ambiguïté de son double objectif. D'une part, réguler l'activité des pêcheurs, qui ne pourront durablement pas jouer sur les deux tableaux : exercer une influence au sein du comité de gestion et contourner les règles de façon permanente. Le PGEM ne pourrait-il pas être plus efficace et légitime si, d'une manière ou d'une autre, les pêcheurs pouvaient y jouer un rôle de régulateurs de leurs propres activités? D'autre part, le PGEM doit pouvoir accompagner et favoriser l'essor des tendances touristiques, tout en contrôlant leur développement pour éviter les excès et les risques de dégradation de l'environnement.

\section{BIBLIOGRAPHIE}

BARÉ Jean-François, 2002. Le Malentendu Pacifique, Paris, éditions Hachette, coll. Histoire des Gens, $280 \mathrm{p}$. 
Blanchet Gilles, 1990. La pêche au grand filet à Tahiti : la tradition à l'épreuve du changement, in Gilles Blanchet (éd.), Les petites activités de pêche dans le Pacifique Sud, Paris, IRD, pp. 185-202.

Bulletin Officiel des Établissements Français d'Océanie (BOEFO), 1897. Papeete, Imprimerie officielle, $363 \mathrm{p}$.

Charles Mahe, 2005. Functions and socio-economic importance of coral reef and lagoon, and implication for sustainable management. Case study of Moorea, French Polynesia, MSc Thesis (Major) Environmental Sciences, Environmental Systems Analysis Group (ESA), Department of Environmental Sciences, Wageningen University, the Netherlands, $159 \mathrm{p}$.

Calinaud N R., 1993. La situation juridique des lagons polynésiens, Bulletin de la Société des études océaniennes 260, Tome XXII, 12, pp. 47-54.

Conte Éric, 1999. L’archéologie en Polynésie française. Esquisse d'un bilan critique, Papeete, Au vent des îles, $302 \mathrm{p}$.

David Gilbert et Aurélie Thomassin, 2007. Les aires marines protégées, petit voyage autour d'un territoire en mal de reconnaissance, Premier colloque national sur les aires marines protégées, Boulognesur-mer, 20-22 novembre 2007, 15 p.

DAvIES John, 1991. A Tahitian and English dictionary,

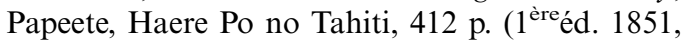
London Missionary Press).

Deleuze Gilles et Félix Guattari, 1980. Mille plateaux, Paris, éd. de Minuit, 645 p.

Di Méo Guy, 1998. De l'espace aux territoires : éléments pour une archéologie des concepts fondamentaux de la géographie, L'information géographique 3, pp. 99-110.

Galzin René, Éric Morize, Arsène SteIn et Éric ConTE, 1989. Dégradations naturelles et/ou anthropiques en zones côtières intertropicales et répercussions possibles sur l'économie des pêches : le cas des récifs coralliens, Action CORDET, Rapport du centre de recherche insulaire et observatoire de l'environnement 32, $135 \mathrm{p}$.

Ghasarian Christian, Tamatoa Bambridge et Philippe GesLIN, 2004. Le Développement en question en Polynésie française, Journal de la Société des Océanistes 119, pp. 211-222.

Gaspar Cécile, Olivier Château et René Galzin, 2008. Feeding sites frequentation by the pink whipray Himantura fai in Moorea (French Polynesia) as determined by acoustic telemetry, Cybium 32 (2), pp. 153-164.

Godelier Maurice, 1984. L'Idéel et le Matériel, Pensées, économies et sociétés, Paris, Fayard, 348 p.

Green Roger C. K., Roy A. Rappaport, A. RapPAPORT et J. DAVIDSON, 1968. Archeology on the island of Mo'orea, French Polynesia, New York, Anthropological Papers of the American Museum of Natural History 51, Part 2.
Institut de la Statistique en Polynésie française (ISPF), 2008. http://www.ispf.pf.

JAussen Tepano, 1996 ( 8 édition). Dictionnaire de la langue tahitienne, Papeete, Société des études océaniennes.

Journal Officiel de la Polynésie française (JOPF), 2004 (22 octobre 2004). Arrêté no 410 CM rendant exécutoire le PGEM de l'île de Moorea.

Jullien-Parra Sylvie, 2007. Madame Bobby, pionnière du tourisme en Polynésie française 1934-1976, Papeete, éd. Le Motu, Collection expression libre, $124 \mathrm{p}$.

MÉtraux Alfred, 1980. L'île de Pâques, Paris, Gallimard.

Morrison James, 1956. Le journal de James Morrison, second maître à bord de la Bounty, traduit par Bernard Jaunez, Paris, Société des Océanistes, Publications de la Société des Océanistes 16, 200 p.

OLIVEr Douglas, 1974. Ancient Tahitian Society, 3 vol. The University Press of Hawaii, Honolulu, Hawaii.

Oracion Enrique, Marc Miller et Patrick Christie, 2005. Marine protected areas for whom? Fisheries, tourism, and solidarity in a Philippine community, Ocean and Coastal Management 48, pp. 393-410.

OттіNo Pierre, 1965. La pêche au grand filet ('upe'a rahi) à Tahiti, cahiers ORSTOM, série Sciences humaines 2, 2, 75 p.

RAFFESTIN Claude, 1986. Écogénèse territoriale et territorialité, in F. Aurillac et R. Brunet, Espaces, jeux et enjeux, Fayard, Paris, pp. 175-185.

Retaillé Denis, 1997. Le monde du géographe, Paris, Presses de Sciences Po.

Robineau Claude, 1984. Tradition et modernité aux îles de la Société. Livre I : Du coprah à l'atome, Paris, éd. de l'orstom, collection Mémoires 100, 489 p.

Rigo Bernard, 2004. Altérité polynésienne ou les métamorphoses de l'espace-temps, Paris, CNRs édition.

SAHLins Marshall, 1989 ( $1^{\mathrm{e}}$ édition en anglais, 1985). Des îles dans l'Histoire, Paris, Hautes étudesGallimard-Le Seuil.

Salvat Bernard, Bertrand Cazalet et François FERAL, 2007. Quelle(s) définition(s) pour les AMP françaises et l'affichage territorial, national et international de leurs surfaces? Réflexions sur les AMP outre-mer, Présentation au $1^{\mathrm{er}}$ colloque national sur les Aires marines protégées, Boulogne sur Mer, 20, 21 et 22 Novembre 2007 et note approfondie dans l'article Courrier de la Nature de juillet 2008.

TE Rangi Hiroa, 1987. The coming of the Maori, Wellington, Whitcoulls.

Te Vea Tautai, 2006 (18 avril). La lettre du ministre de la pêche.

VIEUx Caroline, 2002. La pêche lagonaire à Moorea (Polynésie française): évolution quantitative et socio-économique depuis 1992, rapport pour Ifrecor, Naturalia et Biologia, EPHE, 42 p. 
YONGER Marie, 2002. Approche de la pêcherie récifolagonaire de Moorea (Polynésie française) : évaluation de la production halieutique et de la population des pêcheurs, mémoire de fin d'études pour l'obten- tion du diplôme d'Agronomie approfondie, spécialisation Halieutique, bibliothèque du CRIOBE, Moorea. 
